\title{
DISEÑO DE CURSO VIRTUAL PARA APOYAR EL PROCESO DE ENSEÑANZA APRENDIZAJE DE LA DISCIPLINA DE INGENIERÍA Y GESTIÓN DE SOFTWARE EN LA UNIVERSIDAD DE LAS CIENCIAS INFORMÁTICAS
}

\author{
VIRTUAL COURSE DESING TO SUPPORT THE TEACHING LEARNING \\ PROCESS OF DE DISCIPLINE OF SOFTWARE ENGINEERING AND \\ MANAGEMENT AT THE UNIVERSITY OF COMPUTER SCIENCE
}

\author{
Ailec Granda Dihigo \\ agranda@uci.cu \\ Universidad de las Ciencias Informáticas (Cuba)
}

\section{RESUMEN}

Este articulo muestra las distintas etapas y los resultados correspondientes al diseño, desarrollo e implementación de un curso virtual de Ingeniería de Software, cuyo objetivo era potenciar el desarrollo del proceso de enseñanza-aprendizaje de la Ingeniería de Software en la Universidad. Como referencia se utilizo el modelo ADDIE, que preemitió la evaluación del curso. Los resultados obtenidos certificaron la calidad del producto y el cumplimiento de sus objetivos.

PALABRAS CLAVE: Tecnología de la Información y las Comunicaciones, Ingeniería de Software, Proceso de Enseñanza-Aprendizaje, Entorno Virtual, Curso Virtual.

\begin{abstract}
This article shows the various stages and results for the design, development and implementation of a virtual course of Software Engineering, whose aim was to promote the development of teaching-learning process of Software Engineering at the University. As a reference ADDIE model was used, which allowed the evaluation of the course. Final results certified the quality of the project and compliance with the objectives.
\end{abstract}

KEYWORDS: Communication Information Technology, Software Engineering, TeachingLearning Process, Virtual Environment, Online Course. 


\section{INTRODUCCIÓN}

Actualmente las Tecnologías de la Información y la Comunicación (TICs) se están desarrollando vertiginosamente, lo cual afecta todos los campos de nuestra sociedad, donde la educación no es una excepción. Los rápidos cambios, el aumento de los conocimientos y las demandas de una educación de alto nivel constantemente actualizada se convierten en una exigencia permanente (Uriza, 2010). Las mismas se han convertido en herramientas indispensables para acelerar los procesos de enseñanza - aprendizaje y elevar su calidad, siendo ya una realidad y una necesidad social impuesta por el desarrollo tecnológico alcanzado. El desarrollo de la Educación Superior marcha aparejado a todos estos avances. Las universidades cubanas han adoptado las TIC en su proceso de formación. Como máxima expresión de la ocupación e importancia que le ha dado el país, al desarrollo de las Tecnologías de la Información y las Comunicaciones y de la informática, se crea en el año 2002 la Universidad de las Ciencias Informáticas (UCl), la cual tiene como estrategia, la explotación constante de la tecnología, en función de sus 3 procesos fundamentales: Formación, Producción e Investigación. La disciplina de Ingeniería y Gestión de Software forma parte de la columna vertebral en la formación de los Ingenieros en Ciencias Informáticas de esta universidad. La misma constituye uno de los principales espacios, donde el estudiante puede aplicar en la práctica productiva, el resultado obtenido durante el proceso de enseñanza- aprendizaje.

\section{Orígenes del curso virtual de apoyo a las asignaturas de Ingeniería de Software.}

En los primeros cursos que se impartieron las asignaturas de Ingeniería de Software en la $\mathrm{UCl}$, no existía ningún entorno virtual de aprendizaje (EVA), por lo que el trabajo cooperativo e interactivo en las materias, se hacía muy engorroso, y en ocasiones imposible. Esto implicaba, que no se contara con ningún curso virtual que apoyara la enseñanza-aprendizaje de la IS en la universidad. Ante esta situación, el Departamento Docente Central de Ingeniería y Gestión de Software, el cual se encarga de guiar metodológicamente la asignatura, tuvo que adoptar variantes, que al menos garantizaran la distribución de contenidos y materiales. Se contaba entonces con un sitio Web, en el cual se colgaban las clases de las asignaturas de la disciplina, sin brindar ninguna posibilidad de comunicación virtual entre estudiantes y profesores, ni entre los propios estudiantes.

En el curso 2007-2008, la Dirección de Teleformación, perteneciente a la Vicerrectoría de Formación, establece la utilización del EVA (Moddle) para apoyar el proceso docente educativo de las diferentes disciplinas y asignaturas de la carrera.

Es así como surge en ese año, el curso virtual de Ingeniería de Software, el cual apoyaba el proceso de enseñanza aprendizaje de la asignatura. Este curso, aunque facilitaba mucho más la interacción entre los actores del proceso, tampoco cumplía con las características fundamentales que debe tener un curso de este tipo, pues generalmente la participación de los estudiantes era poca, los materiales y recursos que se publicaban en el mismo, en muchas 
ocasiones no estaban adaptados a este tipo de actividad, por lo que, aunque apoyaba la impartición de la asignatura, realmente no contribuía a potenciar el desarrollo de esta disciplina en la Universidad.

Como se puede ver, se generó una situación problemática puesto que se hizo necesario perfeccionar la disciplina de Ingeniería y Gestión de Software, fundamentada sobre el uso de la tecnología. Era una realidad que en esos momentos no se explotaban todos los recursos tecnológicos de los cuales se disponía. La universidad contaba con un grupo de recursos, que en la mayoría de los casos, no se utilizaban o simplemente se usaban en otros fines, para los cuales no fueron diseñados. Todo esto provocó que el proceso no se desarrollara con la rapidez y calidad que requería y que no se lograra crear las habilidades y competencias que brinda la disciplina y que necesita un graduado de esta universidad.

\section{Las TIC en la enseñanza de la Ingeniería de Software.}

Los métodos de enseñanza de la Ingeniería de Software han progresado, a la par de que han sido utilizados por otras didácticas particulares, desde una enseñanza tradicional y clásica a, basada en casos, luego basada en problemas y por último, siendo la tendencia más actual a la enseñanza, basada en proyectos. Esa tendencia no solo ocurre en el ámbito nacional, sino también en el internacional (Ciudad, 2009).

La enseñanza de esta Disciplina, no ha estado ajena a la introducción de las nuevas tecnologías, por lo que este hecho ha traído un grupo de ventajas al proceso, en el cual se ha beneficiado el alumnado que recibe esta disciplina. La posibilidad de interacción constante entre los propios estudiantes, ha propiciado un reforzamiento en el trabajo en equipo, lo cual es muy importante en este tipo de ingeniería. Además, los alumnos emprenden una búsqueda y replanteamiento continuo de contenidos y procedimientos, aumentando la implicación en sus tareas y el desarrollo de iniciativas.

Es importante destacar, que el uso de las TIC en el proceso de enseñanza aprendizaje de la Ingeniería de Software, favorece el trabajo colaborativo. El intercambio de ideas, la discusión y la posibilidad de toma de decisiones, contribuye sin dudas a un mejor desarrollo de las habilidades y competencias requeridas. La posibilidad de compartir, aplicar y gestionar el conocimiento a través de entornos virtuales, proporcionados por el desarrollo de las TIC, ha constituido uno de los principales aportes a la enseñanza de esta Disciplina. 


\section{METODOLOGÍA}

En función de dar respuesta a la problemática `planteada, y teniendo en cuenta el análisis de las potencialidades que brindan las nuevas tecnologías a la enseñanza de la Ingeniería de Software, nos dimos a la tarea de diseñar un curso virtual en un EVA para desarrollar el proceso de enseñanza-aprendizaje de la disciplina de Ingeniería y Gestión de Software en la Carrera "Ingeniería en Ciencias Informáticas". Para ello se contó con la colaboración de todos los profesores de la disciplina.

Para el diseño del curso, se trabajó con la modalidad de educación presencial apoyada por las TIC, por el hecho de que en el contexto donde se aplicaría, existen un grupo de restricciones en cuanto al cumplimiento del plan de estudios de la carrera y el programa analítico de las asignaturas. Además, el estudio realizado sobre estos temas, nos permitió considerar que aunque algunos especialistas han definido que esta modalidad ocurre cuando los profesores y estudiantes comparten el mismo espacio y el mismo tiempo, utilizando las TIC como medio, como recurso o como método en el aprendizaje de los estudiantes; se pueden diseñar cursos y materiales didácticos, que sirvan de apoyo a la actividad presencial, y que no sea obligatorio encontrarse en el mismo lugar y momento para el desarrollo de las diferentes actividades. Se debe destacar además, que esta modalidad, tiene un grupo de características que permiten flexibilizar y personalizar el trabajo tanto de estudiantes como de los profesores.

Teniendo en cuenta los resultados de estudios comparativos realizados por otros autores, el análisis de las principales características de la plataforma Moodle, las ventajas que ella proporciona, el hecho de que cumple con las condiciones pedagógicas, tecnológicas y organizativas que debe presentar una plataforma de este tipo según De Benito (2006) y tomando en consideración que en la Universidad de las Ciencias Informáticas se utiliza este EVA; se decidió que el curso que se obtendría como resultado del proyecto, fuera montado en la plataforma Moodle.

Durante el desarrollo de la investigación, se combinaron técnicas cuantitativas y cualitativas. La metodología cualitativa utilizada fue la investigación-acción, por considerarse la más adecuada al contexto y a las características del proyecto. En el caso de las cuantitativas, se utilizó un diseño cuantitativo descriptivo, específicamente se utilizó como aproximación metodológica la encuesta, diseñándose 3 cuestionarios para su aplicación.

\section{Modelo de diseño instruccional utilizado.}

Para el diseño del curso, se utilizó el modelo de diseño instruccional ADDIE, desarrollándose las 5 etapas que propone: Análisis, Diseño, Desarrollo, Implementación y Evaluación. En el caso de la última etapa, se realizaron los 2 tipos de evaluaciones, tanto formativa como sumativa. En el caso de la formativa, se fue aplicando durante todo el desarrollo del curso, con el objetivo de verificar los logros que se iban obteniendo e ir corrigiendo los problemas que se fueran detectando. 
Al final, se desarrolló una evaluación sumativa, teniéndose en cuenta el criterio de los usuarios. Para esto se aplicó el modelo propuesto por Kirkpatrick el cual establece las bases de la evaluación de la formación continua.

Los cuatro niveles del modelo de evaluación de Kirkpatrick son: (Pineda, 2000)

I. Reacción de los participantes ante la formación, es decir, nivel de satisfacción con la formación recibida (Reacción).

II. Aprendizaje realizado por los participantes o nuevas competencias adquiridas gracias a la formación (Aprendizaje).

III. Conducta de los participantes en el puesto de trabajo, es decir, transferencia de los aprendizajes realizados al propio puesto (Comportamiento).

IV. Resultados en la organización, es decir, efectos que la formación genera en las diferentes áreas de la organización (Resultados).

Para la aplicación de los diferentes niveles, se diseñaron 3 encuestas. En el caso del nivel de reacción, se aplicó un cuestionario, para medir el grado de satisfacción del alumnado con el desarrollo de la asignatura y su interacción con la tecnología. Este instrumento también contenía preguntas relacionadas con el nivel de comportamiento.

Para el nivel de aprendizaje, se tomó como indicadores de la evaluación, los resultados docentes, siendo comparados con los resultados del curso anterior, en el cual no utilizaron el curso diseñado.

Para el nivel de comportamiento, se realizó además, un cuestionario con el objetivo de medir la aplicación de lo aprendido por los estudiantes del 3er año que recibieron la asignatura de Ingeniería de Software, en los proyectos productivos en los cuales trabajan.

Para el nivel de resultados, se aplicó otro instrumento a un grupo de especialistas en el área de la Ingeniería y Gestión de Software, donde se midió el impacto de la formación recibida por los alumnos, en la organización.

Se aplicó además, un cuestionario a 6 expertos en el tema, para corroborar la validez del diseño del instrumento utilizado en el nivel de reacción. A partir de las recomendaciones y sugerencias expuestas, se realizaron algunos cambios a la propuesta inicial, culminando el diseño del cuestionario a aplicar.

\section{Población y muestra}

La población que utilizó el curso virtual propuesto, fueron los 1498 estudiantes del 3er año de la carrera de Ingeniería en Ciencias Informáticas en la $\mathrm{UCl}$, los cuales recibieron en ese momento la asignatura de Ingeniería de Software.

Para la aplicación del instrumento que medía el grado de satisfacción de los estudiantes con el 
curso, se tomó una muestra probabilística estratificada de 150 estudiantes de la población definida.

\section{RESULTADOS}

En este apartado, presentaremos el desarrollo de las diferentes fases en todo el proceso de creación del curso, apoyado para ello en el modelo de diseño instruccional ADDIE.

\section{Análisis de la Situación Problémica}

Se comenzó realizando un análisis exhaustivo del proceso de enseñanza aprendizaje de la Disciplina de Ingeniería y Gestión de Software en la UCI. Posteriormente, se procedió a analizar si la utilización de las TIC podría contribuir al desarrollo del proceso. Como resultado del estudio, se determinó perfeccionar la disciplina, sustentada en el uso de las nuevas tecnologías como eje central, aprobándose la idea de diseñar e implementar el curso virtual.

\section{Diseño del Curso Virtual de Ingeniería de Software}

En esta fase se llevó a cabo el diseño del curso virtual estableciendo las características, herramientas, recursos y actividades de las cuales debía disponer. Se estableció además el rol que debían jugar tanto los estudiantes como los profesores.

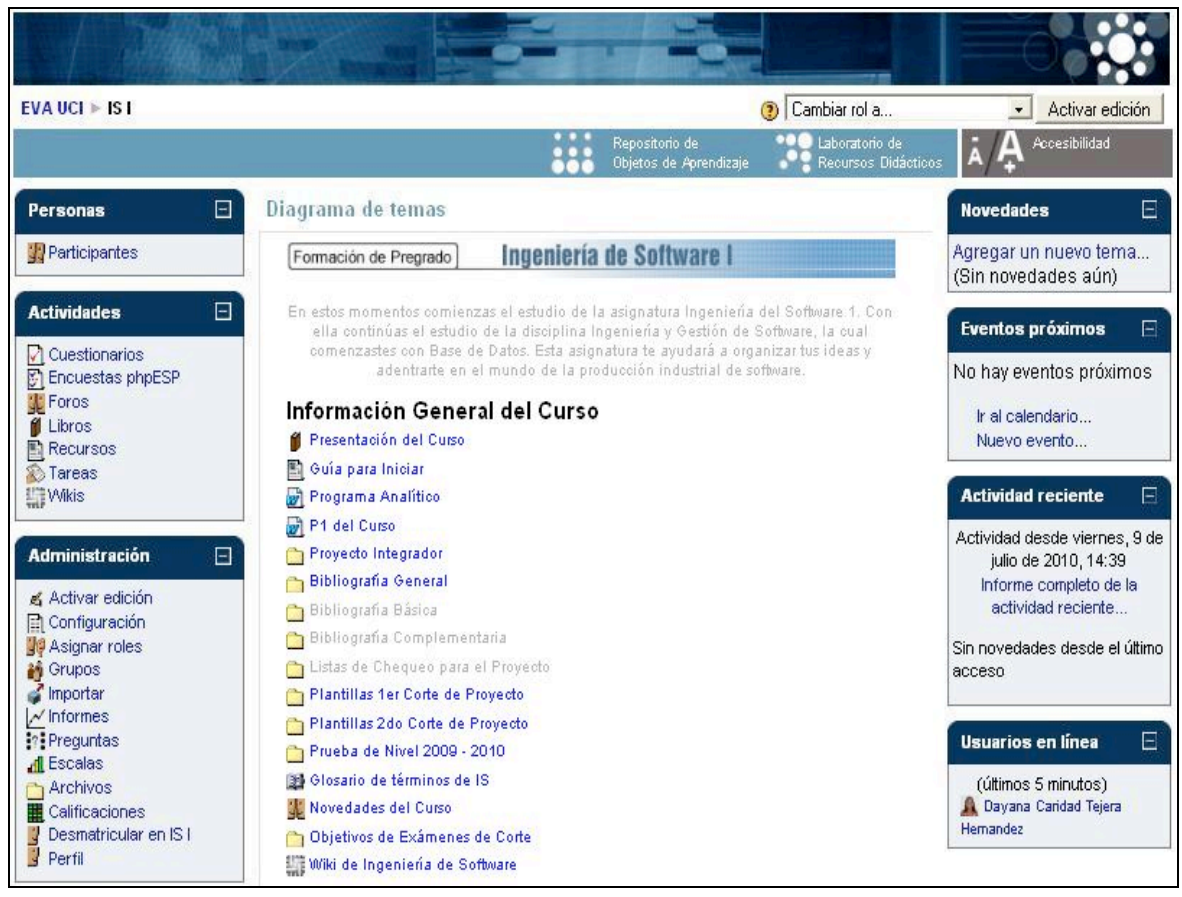

Figura 1. Pantalla General del Curso 


\section{Organización del contenido.}

El contenido del curso virtual se concibió como un complemento de la formación presencial, donde se hizo más hincapié en las actividades y ejercicios, así como en la información adicional de interés para los estudiantes. No obstante, se buscó mantener el contacto con los alumnos fuera del aula de clases y de su horario habitual. La distribución del mismo se realizó según los temas y semanas del curso presencial.

En cada una de las semanas, se visualizaba el tema, objetivos y materiales de la actividad presencial, así como las características y recursos necesarios para la realización de las actividades diseñadas para el desarrollo del estudiante en modalidad no presencial. Se puso a disposición del alumnado materiales básicos para el desarrollo de la actividad presencial, los cuales podían ser accedidos y estudiados antes de dichos encuentros. Se publicaron además, materiales complementarios, dedicados a la actividad fuera del aula y de auto-estudio de los alumnos. Ver Figura 2.

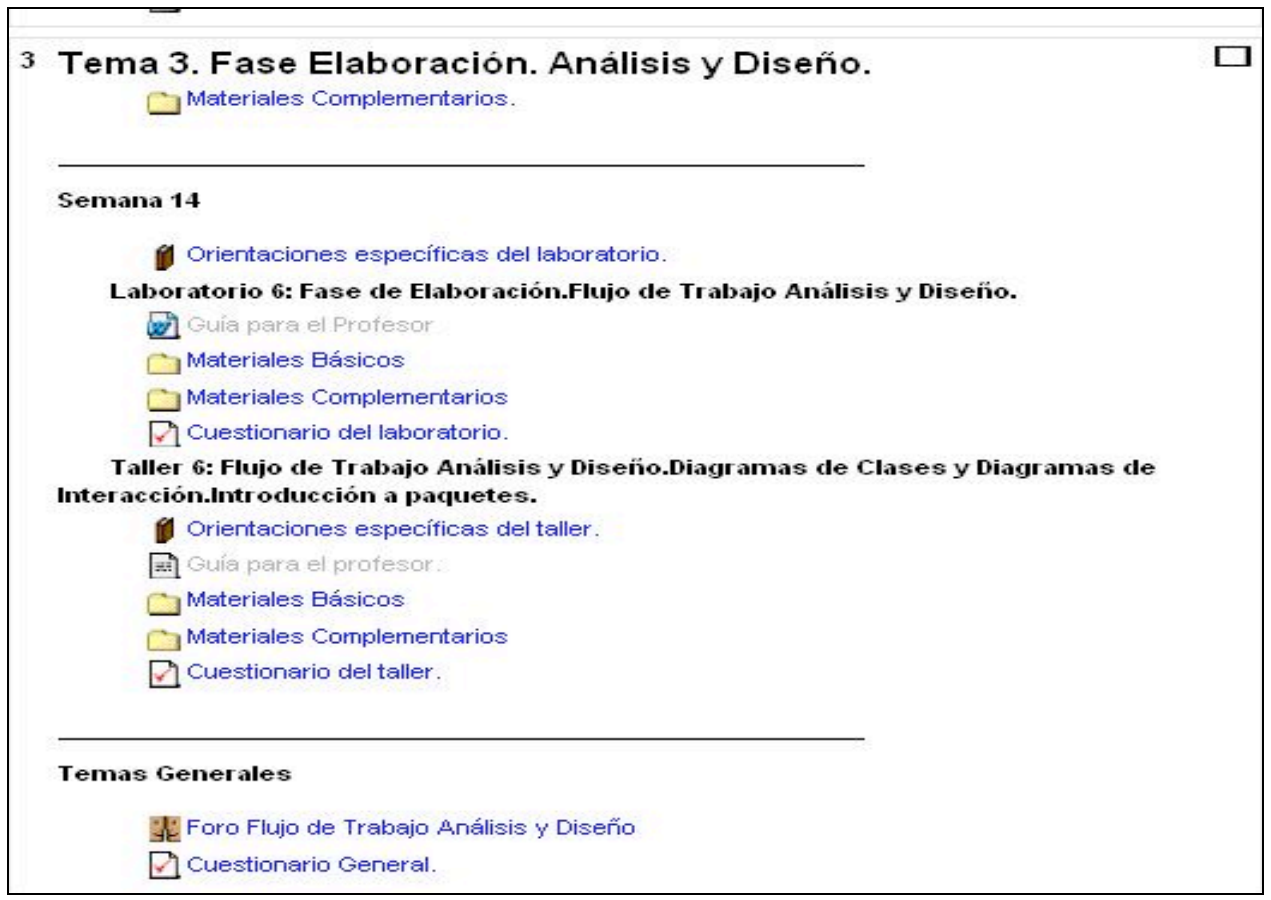

Figura 2. Distribución de Contenidos por Tema.

\section{Tipos de recursos y actividades.}

Para el diseño del curso se utilizaron los siguientes espacios:

- Espacios permanentes, relacionados con el proceso. Se estableció la comunicación social entre estudiantes-estudiantes, profesor-estudiantes y profesor-profesor.

- Espacios puntuales relacionados con los contenidos del curso, los cuales tuvieron una temporalización determinada, por lo que se hicieron visibles al iniciarse el período 
habilitado y quedaron cerrados al finalizar.

\section{Herramientas de Comunicación utilizadas}

- Foros Generales: Se utilizaron para exponer cuestiones novedosas de la disciplina. Ver Figura 3

- Foros destinados al trabajo grupal: Se activaron foros en cada tema, utilizándose para reflexionar sobre temas específicos de la asignatura. También se aclararon dudas sobre contenidos específicos.

Cada uno de los foros habilitados, explicaban la temática a debatir y objetivos del mismo, con el fin de incrementar su funcionalidad.

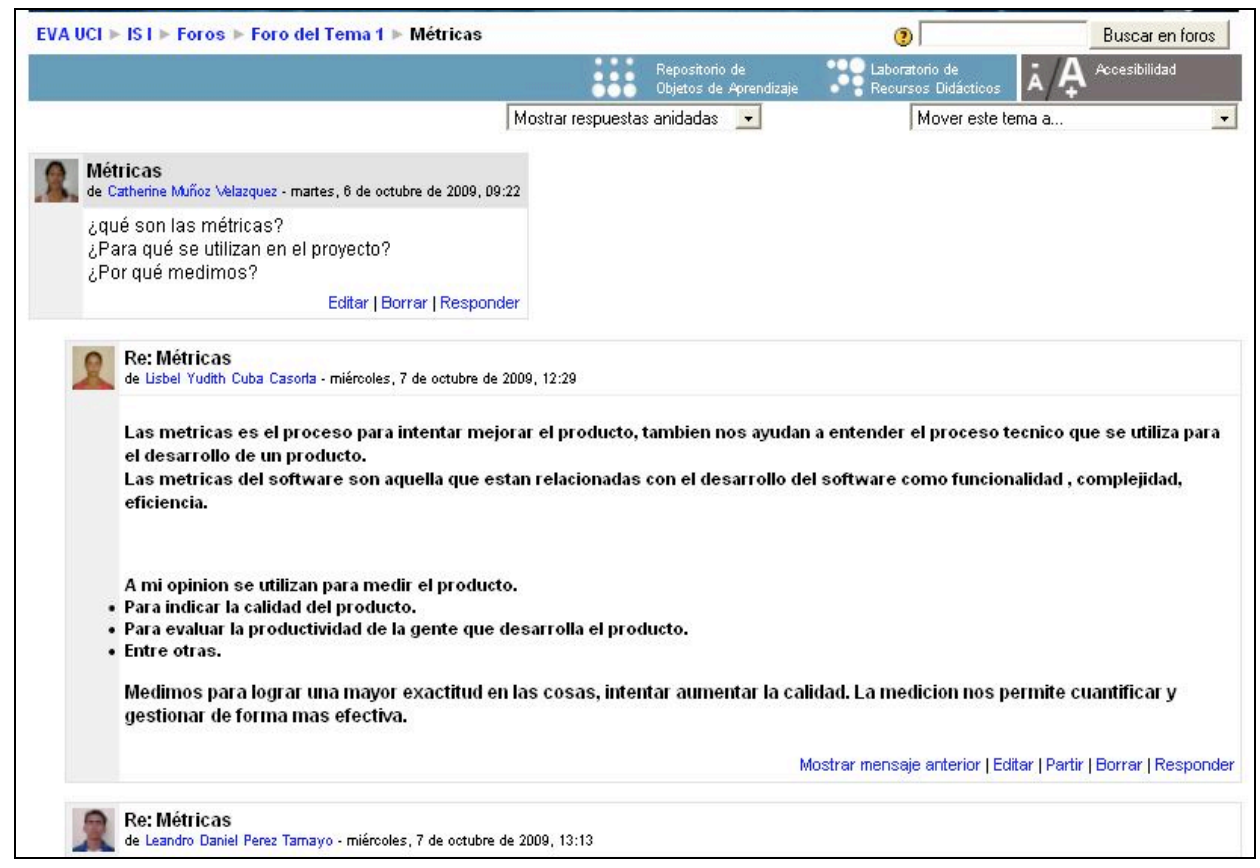

Figura 3. Ejemplo de debate en el Foro

\section{Herramientas de Trabajo Colaborativo utilizadas}

- Glosarios: Se utilizó para reflejar el significado de los términos fundamentales que se trabajaron durante el desarrollo de la asignatura. En el mismo tanto los estudiantes como los profesores tenían la posibilidad de incluir y modificar términos, de forma que se lograra unificar el vocabulario utilizado en la asignatura. La participación de los alumnos en esta actividad, era seguida por el profesor, para evitar que se introdujera información o datos inconsistentes.

- Recurso Libros: Se utilizó para presentar las características y objetivos de la asignatura, así como de cada uno de los temas y actividades. Los profesores también tenían la posibilidad de modificar este recurso, con el objetivo de aportar sus ideas y experiencias.

- Recurso Carpeta: Se utilizó para organizar la documentación disponible en el curso. Los 
diferentes materiales, tanto básicos, como complementarios, se ubicaron dentro de carpetas y subcarpetas. Los profesores y estudiantes tenían la posibilidad de subir nuevos archivos, aunque en el caso de los estudiantes, debían consultarlo previamente con el profesor, para así poder garantizar la veracidad de la información.

- Enlaces a Web y otros archivos: Se utilizó para poder acceder a páginas Web y otros documentos relacionados con diferentes temas de la Ingeniería de Software. Todos los usuarios tenían la posibilidad de añadir o comentar los enlaces existentes

- Wiki: Se utilizó para que tanto profesores como alumnos pudieran poner a disposición del resto, diferentes materiales, reflexiones y vínculos, donde se trataran temas de la Ingeniería de Software.

Para la auto-evaluación y evaluación en los diferentes espacios del curso, se utilizaron fundamentalmente las siguientes actividades:

- Cuestionarios: Se utilizaron para medir los conocimientos en cada una de las actividades de la asignatura. Se realizaron además, cuestionarios generales, de cierre del tema y otros para aplicar como Prueba Parcial. Ver Figura 5

- Tareas: Se utilizó para que los estudiantes realizaran la entrega de algunas actividades en las cuales debían enviar ficheros con la respuesta. Esto se utilizó en temas o actividades específicas.

- Taller: Se utilizó para la exposición de ideas sobre temas específicos de la asignatura. En ocasiones fueron evaluativos.

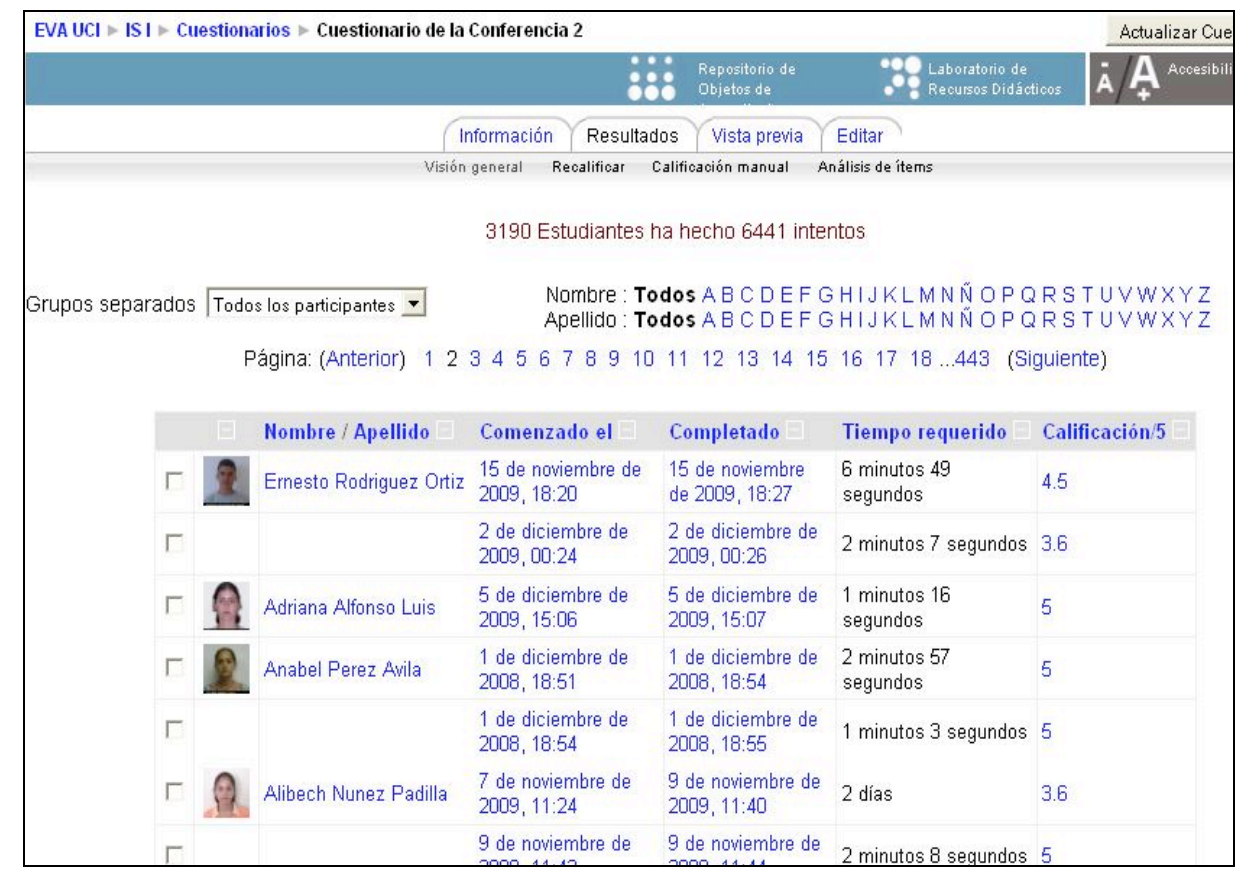

Figura 4. Respuesta a Cuestionario. 


\section{Rol del profesor}

El curso se diseñó como complemento al proceso de aprendizaje, por lo que se definió que se trabajaran dos roles diferentes de profesor:

- Profesor - Autor: Aquel que gestionaría los contenidos a ser trabajados en el curso, diseñando las actividades de aprendizaje y dando las pautas para la evaluación de los procesos.

- Profesor - Tutor: El que establecería una comunicación directa con el estudiante a través de las diferentes herramientas disponibles en el curso.

Tomando en cuenta que la tutoría es esencial para el logro de los objetivos propuestos en el curso, los profesores-tutores, debían tener entre sus funciones las siguientes tareas:

- Facilitador del proceso de aprendizaje.

- Motivar al alumno a participar en el curso.

- Mantener una comunicación con el alumno.

- Evaluación.

- Apoyo técnico.

\section{Rol del alumno}

Durante el desarrollo del curso, los estudiantes debían lograr:

1. Adquirir autonomía con el conocimiento.

2. Conocer las herramientas indispensables para utilizar en cursos virtuales.

3. Participar en las diferentes actividades propuestas, realizando tanto trabajo individual como en equipo.

4. Plantear a sus compañeros y al profesor las dificultades y dudas que se le presentaban a partir de la realización de las actividades, o a partir de algún tema de debate expuesto en los foros de discusión.

5. Formar grupos de trabajo con sus compañeros, para la realización de trabajo colaborativo.

6. Planificar bien su tiempo, de manera que pudieran cumplir el cronograma del curso.

\section{Desarrollo}

En esta etapa se elaboraron los contenidos, actividades, evaluación, así como los diferentes materiales que debían utilizar los estudiantes, creándose el ambiente de aprendizaje necesario para lograr los objetivos trazados con dicho curso.

Una vez elaborados todos lo materiales necesarios para poner a disposición de los estudiantes, se procedió al montaje del curso en el EVA Moodle (el cual se utiliza en la universidad). Este 
proceso se desarrolló en 2 meses, aunque durante su utilización, también se iban desarrollando algunos cambios, a partir de la identificación de algunos problemas, resultado de la evaluación formativa que se realizaba.

\section{Implementación}

En esta etapa, se puso a disposición de toda la comunidad el curso. El mismo comenzó a ser utilizado por los 1498 estudiantes del 3er año de la carrera Ingeniería en Ciencias Informáticas.

La participación en las diferentes herramientas de comunicación disponibles, fue buena. Estos espacios, además de utilizarse para la aclaración de dudas y debate, sirvieron para que el profesor tuviera una medida de cómo iban sus estudiantes, incluyendo este elemento en los criterios para la evaluación.

\section{Evaluación (Análisis de los resultados)}

Como se explicó anteriormente, para la evaluación del curso, se aplicaron los dos tipos de evaluación, formativa y sumativa. Es por ello que se aplicaron 3 instrumentos a estudiantes, profesores y especialistas, con el objetivo de abarcar los 4 niveles que se abordan en el modelo Kirpatrick.

Para el procesamiento de los datos, se procedió a codificar y transferir la información a una matriz en una hoja Excel. En la misma se procesaron todo los datos obtenidos a partir del los instrumentos aplicados.

Para el primer nivel, se aplicó el cuestionario a una muestra de los estudiantes que utilizaron el curso. Los resultados reflejaron el alto grado de satisfacción de dichos estudiantes, en cuanto a: habilidades desarrolladas, conocimientos básicos, atención al alumnado, proceso de enseñanza aprendizaje, organización de la enseñanza, infraestructura para el proceso formativo, aporte de las TIC a la asignatura y la aplicación en la práctica laboral de los conocimientos adquiridos.

En el caso del segundo nivel, se analizaron los resultados docentes obtenidos por los estudiantes que trabajaron con el curso. Se evidenció que los resultados obtenidos por los estudiantes fueron mejorando paulatinamente, hasta llegar al más alto en este año, con un 95,59\% de promoción, comparándolo con un 93.16 porciento obtenido en el curso anterior. Ver Figura 6. 


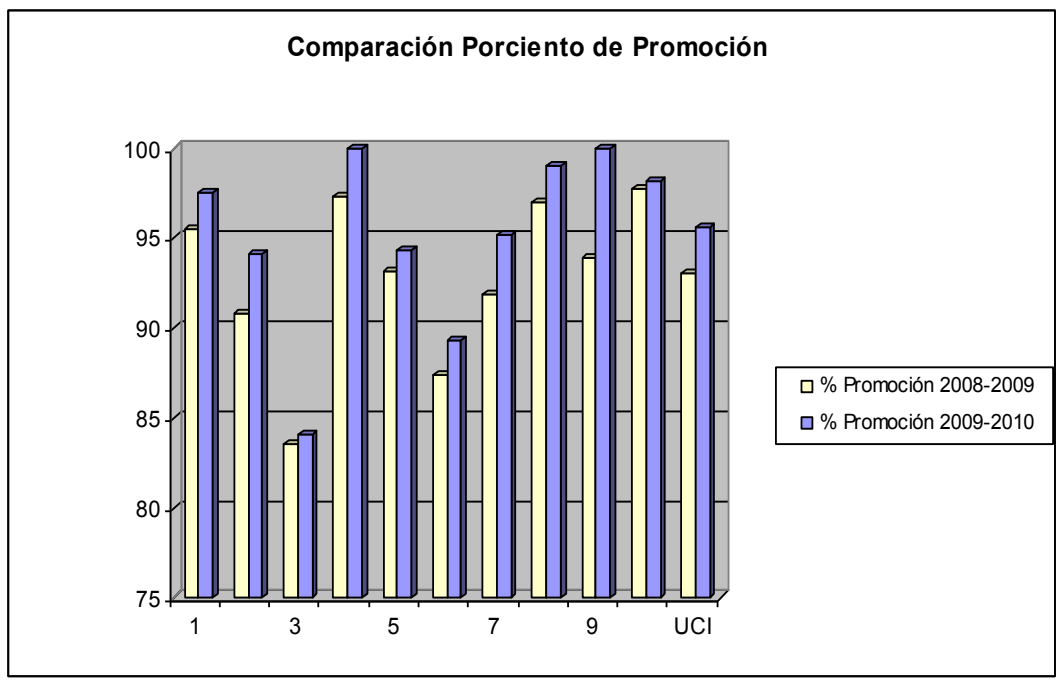

Figura 5. Gráfico comparativo de Porciento de Promoción.

La calidad de los resultados también mejoró, donde más del $50 \%$ de los alumnos obtuvieron calificaciones entre excelente y bien, mientras en el curso anterior, solo se llegó a un $48.14 \%$.

Para el nivel 3, se aplicó un cuestionario a los profesores de Ingeniería de Software que trabajan en los proyectos productivos junto a los estudiantes de 3er año. La información obtenida tras su aplicación evidencia que la asignatura está concebida correctamente, de forma que los contenidos que se imparten en ella se relacionan estrechamente con el trabajo que deben realizar los estudiantes en los proyectos productivos. Se demostró que el curso fue bien diseñado, de manera que se logró la transferencia de lo aprendido a la actividad laboral, que en nuestro caso es el trabajo diario en el proyecto productivo. Ver Figura 7.

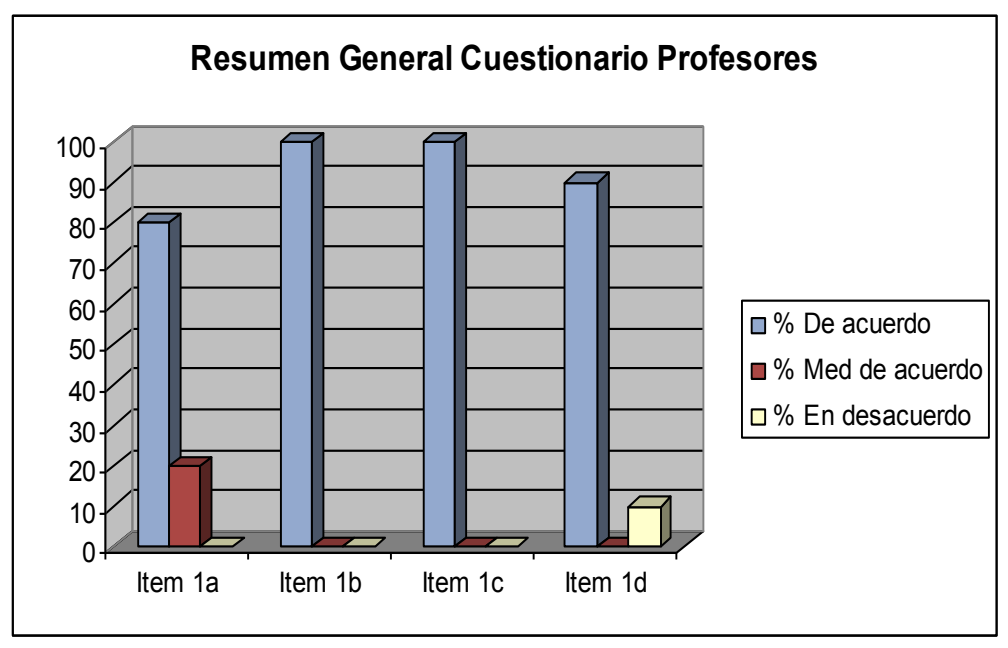

Figura 6. Cuestionario Profesores. Resumen General.

Para el nivel de resultados (4), se aplicó un cuestionario a un grupo de especialistas en el área de la Ingeniería y Gestión de Software. De la información obtenida en este instrumento, se aprecia que en sentido general la formación de los estudiantes que recibieron la asignatura con la utilización del curso virtual, fue mucho mejor, reflejándose directamente al incorporarse a los 
proyectos. La calidad del trabajo realizado en dichos proyectos, evidencian que la formación estuvo muy bien concebida y desarrollada como proceso. La institución se ha beneficiado con estos cambios, pues contribuye directamente a desplegar su proceso productivo. Ver Figura 8.

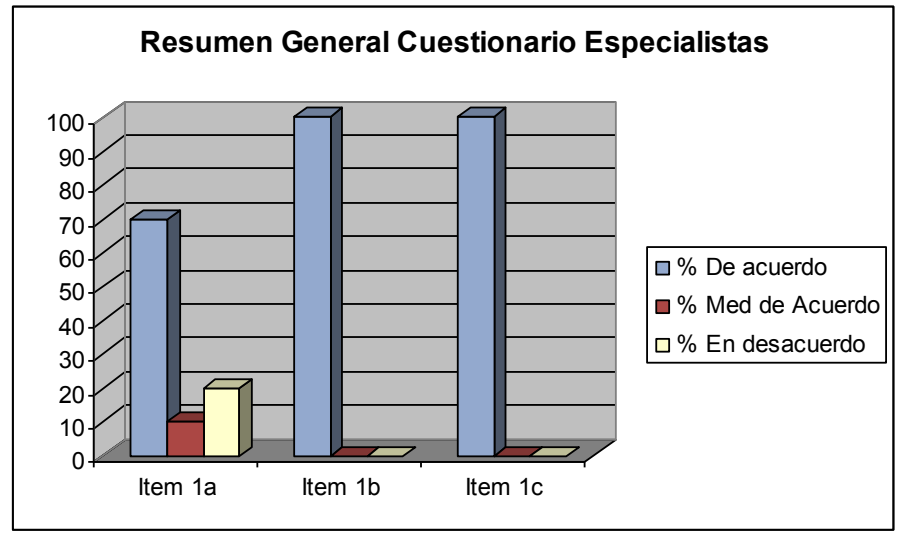

Figura 7. Cuestionario Especialistas. Resumen general.

En el caso de la evaluación formativa, se realizaba frecuentemente, una revisión de la participación de los alumnos en las diferentes actividades, reflejándose en varias ocasiones las insatisfacciones y cuestiones que no compartían. Estas pudieron ser mejoradas en el transcurso del período de utilización del curso.

En sentido general consideramos que el curso fue muy bien aceptado por los estudiantes, reflejándose en su alto nivel de satisfacción, con el uso del mismo. Se pudo apreciar además que sus resultados docentes fueron mejores y que la aplicación en la práctica de lo aprendido fue efectiva, teniendo esto un gran impacto en la Universidad. Después de haber analizado todos los elementos mencionados, podemos afirmar que el curso fue diseñado e implementado exitosamente y que logró potenciar el desarrollo del proceso de enseñanza-aprendizaje de la Ingeniería de Software en la Universidad de las Ciencias Informáticas.

\section{CONCLUSIONES}

Consideramos que el producto obtenido se adapta exitosamente al contexto en el cual está enmarcado. Hablamos de una universidad diferente, donde se estudia solo la Carrera de Ingeniería en Ciencias Informáticas, donde la Disciplina de Ingeniería y Gestión de Software constituye la columna vertebral en la formación de los ingenieros y donde se disponen de una serie de recursos tecnológicos que, aunque en ocasiones fallan, generalmente hacen posible la realización exitosa de las diferentes actividades sustentadas en el uso de las TIC. Los alumnos de esta institución tienen en sus manos todas las posibilidades para explotar el curso, en aras de su formación profesional. Es por ello que para su diseño, se tuvo en cuenta que no les resultaría difícil a los alumnos poder acceder a él, no existiendo grandes limitaciones en cuanto a lugar y hora. 
Podemos decir además, que las características del curso responden a las necesidades formativas del centro, viéndose reflejado, en los resultados obtenidos por los estudiantes, en las habilidades adquiridas, en el nivel de interacción alcanzado y en su preparación para insertarse en la vida productiva.

Los resultados expuestos a partir del procesamiento de los datos obtenidos en los diferentes instrumentos, pudieron reflejar una buena aceptación del curso diseñado, tanto por los estudiantes como por los profesores. La mayoría estuvieron de acuerdo en afirmar, entre otra cosas, que el curso estaba bien estructurado, que permitía la creación de habilidades en el uso de las TIC y que permitía que los contenidos tratados se aplicaran en la práctica laboral.

No obstante, tanto de las encuestas aplicadas, como de la observación de los resultados docentes y de la participación de los estudiantes en las diferentes actividades del curso, se evidencia que algunos no estuvieron suficientemente implicados. Esto provocó que su rendimiento académico en la asignatura fuera más bajo que el del resto de los alumnos y que su desempeño en la producción no fuera adecuado. Consideramos que la causa de esta baja participación, se debe a que algunos estudiantes no se sienten responsables de su proceso de aprendizaje, por lo que no le dan la importancia requerida a estos aspectos, debiéndose considerar la idea de tratar de motivarlos a partir de la dinamización e interactividad que logremos en el curso.

Otro aspecto que es importante destacar, son los problemas tecnológicos que en varias ocasiones afectaron el desarrollo de algunas actividades del curso. Esto limitó de alguna forma la participación, pues en ocasiones el estudiante se desmotivaba al tener problemas para la utilización de algún recurso o desarrollo de alguna actividad. En función de esto, consideramos que la universidad debe garantizar la tecnología necesaria y disponer de todos los especialistas y recursos para resolver cualquier falla tecnológica que se pueda ocasionar.

Una vez desarrollada la evaluación del curso y analizado los elementos expuestos, podemos plantear un grupo de acciones que contribuirán a mejorar y aumentar la incidencia positiva del curso virtual en el entorno. Entre ellas podemos mencionar:

- Mejorar la presentación general del curso, creando un foro para la presentación de los alumnos. En nuestro caso no lo consideramos necesario, puesto que los alumnos ya se conocían en el aula, pero pudiéramos abrir esa posibilidad, de manera que los estudiantes de diferentes grupos puedan conocerse entre sí.

- Crear espacios de Chat para la comunicación en tiempo real entre alumnos y profesores.

- Diseñar nuevas actividades para implicar más a los estudiantes, aumentando la dinámica del trabajo en grupo, la participación en los diferentes espacios disponibles y tratando de enfocarlas mucho más a la utilización de bibliografía, tanto básica, como complementaria.

- Permitir al profesor personalizar las diferentes actividades a partir de las características de sus alumnos.

- Diseñar actividades en el entorno, donde el estudiante requiera de la utilización de otras herramientas informáticas. 
Aunque existieron algunas dificultades (explicadas anteriormente), en sentido general el curso estuvo muy bien pensado, elaborado y ejecutado. Los alumnos estuvieron satisfechos, culminaron la asignatura con un nivel de preparación más alto que en cursos anteriores y se pudo transferir el conocimiento adquirido a la práctica laboral. Estos elementos son determinantes para obtener un curso de alta calidad. El hecho de que tanto los profesores como especialistas pudieran corroborar que los proyectos productivos se beneficiaron con la inserción de los estudiantes de 3er año, y que sus conocimientos en Ingeniería de Software fueron decisivos para el buen funcionamiento y desempeño de los alumnos en su rol, permiten afirmar que realmente el curso diseñado cumplió con su objetivo fundamental: Desarrollar el proceso de enseñanza-aprendizaje de la Ingeniería de Software en la Universidad de las Ciencias Informática.

\section{BIBLIOGRAFÍA}

ÁLVAREZ, JOSÉ V. (2004), "Uso de estándares e-learning en espacios educativos", Universidad Metropolitana de Caracas, Caracas, Venezuela. [en línea]. Disponible en: http://institucional.us.es/revistas/revistas/fuente/pdf/numeros/5/08\%20uso\%20de\%20estand ares.pdf (Consultado Junio 2010)

BARTOLOMÉ, ANTONIO (2001). Universidades en la Red. ¿Universidad presencial o virtual? En Crítica, LII (num. 896) pp. 34-38. [En línea]. Disponible en: http://www.Imi.ub.es/personal/bartolome/articuloshtml/bartolomeSPcritica02.pdf

(Consultado Julio 2010)

CABERO, JULIO; CASTAÑO, CARLOS; CEBREIRO, BEATRIZ; GISBERT, MERCÉ; MARTÍNEZ SANCHEZ, FRANCISCO; MORALES LOZANO, JUAN; PRENDES, M. PAZ; ROMERO, ROSALÍA y SALINAS, JESÚS (2003). Las nuevas tecnologías en la actividad universitaria. Pixel-Bit: Revista de medios y educación, ISSN 1133-8482, №. 20, pags. 81-100

CABERO, JULIO (2000). La formación virtual: principios, bases y preocupaciones, en PÉREZ, R. (coord.): Redes, multimedia y diseños virtuales. Oviedo. Departamento de Ciencias de la Educación, 83-102.

CIUDAD, FEBE A. (2009). Propuesta de perfeccionamiento en la enseñanza de la ingeniería de software en la Universidad de las Ciencias Informáticas, Ciudad de la Habana (inédito).

DE BENITO, BÁRBARA (2006). Diseño y validación de un instrumento de selección de herramientas para entornos virtuales basado en la toma de decisiones multicriterios. Tesis Doctoral. Universidad de las Islas Baleares. Facultad de Educación. Departamento de Pedagogía Aplicada y Psicología de la Educación

GISBERT, M.; ADELL, J.; RALLO, R. \& BELLVER, A. ,(1998), “Entornos Virtuales de EnseñanzaAprendizaje", Cuadernos de Documentación Multimedia, Madrid, Pp. 29-41, [en línea], Disponible en: http://www.ucm.es/info/multidoc/multidoc/revista

MCGRIFF, STEVEN J. (2000). Modelo ADDIE. Instructional Systems, College of Education, Penn 
State University [En línea] $\quad$ Disponible http://disenoinstrucional.files.wordpress.com/2007/09/addiemodel.doc.

PÉREZ SERRANO, GLORIA (2007). Investigación cualitativa. Retos e Interrogantes. Tomo 1. 4ta edición: Editorial La Muralla.

PÉREZ SERRANO, GLORIA (2007). Investigación cualitativa. Retos e Interrogantes. Tomo 2. 4ta edición: Editorial La Muralla.

PINEDA, PILAR (2000). Evaluación del impacto de la formación en las organizaciones. Educar 27, Pág 119-133. Disponible en: http://ddd.uab.cat/pub/educar/0211819Xn27p119.pdf (Consultado Mayo 2010)

ROMERO TOVAR. A (2006). Moodle, Unimos Mentes, Creamos Conocimiento Libre. Ponencia presentada al VI Congreso Internacional Virtual de Educación CIVE 2006, España.

SALINAS, JESÚS (2004). "Innovación docente y uso de las TIC en la enseñanza universitaria". Revista de Universidad y Sociedad del Conocimiento (RUSC). UOC. Vol. 1, no 1. [ En línea] Disponible en: http://www.uoc.edu/rusc/dt/esp/salinas1104.pdf (Consultado Julio 2010].

URIZA, TERESA (2010). Las Tics. Diapositavas de Investigación. Bogota DC, Colombia. [En línea] Disponible en http://www.slideshare.net/theresithaza/diapositivas-de-las-tics-teresa-urizariobueno

\section{Para citar este artículo:}

GRANDA, A. (2010) "Diseño de curso virtual para apoyar el proceso de enseñanza aprendizaje de la disciplina d ingeniería y gestión de software en la universidad de las ciencias informáticas» [artículo en línea]. EDUTEC, Revista Electrónica de Tecnología Educativa. Núm. 34 / Diciembre 2010. [Fecha de consulta: dd/mm/aa]. http://edutec.rediris.es/revelec2/revelec34 ISSN 1135-9250. 